\title{
A MULTI-SCALE MODEL QUANTIFIES THE IMPACT OF LIMITED MOVEMENT OF THE POPULATION AND MANDATORY WEARING OF FACE MASKS IN CONTAINING THE COVID-19 EPIDEMIC IN MOROCCO
}

\author{
Anass Bouchnita* and Aissam Jebrane
}

\begin{abstract}
The coronavirus disease (COVID-19) pandemic emerged in Wuhan, China, in December 2019 and caused a serious threat to global public health. In Morocco, the first confirmed COVID-19 case was reported on March 2, 2020. Since then, several non-pharmaceutical interventions were used to slow down the spread of the disease. In this work, we use a previously developed multi-scale model of COVID-19 transmission dynamics to quantify the effects of restricting population movement and wearing face masks on disease spread in Morocco. In this model, individuals are represented as agents that move, become infected, transmit the disease, develop symptoms, go into quarantine, die by the disease, or become immunized. We describe the movement of agents using a social force model and we consider both modes of direct and indirect transmission. We use the model to simulate the impact of restricting the movement of the population movement and mandating the wearing of masks on the spread of COVID-19. The model predicts that adopting these two measures would reduce the total number of cases by $64 \%$. Furthermore, the relative incidence of indirect transmission increases when control measures are adopted.
\end{abstract}

Mathematics Subject Classification. 35L05, 35L70.

Received April 23, 2020. Accepted April 26, 2020.

\section{INTRODUCTION}

Severe acute respiratory syndrome coronavirus 2 (SARS-CoV-2) is a highly transmissible virus that emerged in Wuhan, China, in December 2019 [10]. Since then, coronavirus disease (COVID-19) has infected more than two million individual around the world, as of April 15 2020. Infectious individuals secrete droplets that contain the virus during coughs and sneezes. As a result, susceptible individuals can directly inhale these droplets during close contact with infectious persons. These droplets also contaminate hard surfaces and can cause the subsequent infection of susceptible individuals. This mode of indirect transmission is important in the case of COVID-19 because stable SARS-CoV-2 can remain for a few days on some type of hard surfaces like plastic and stainless steel [19].

In the absence of effective vaccines or therapeutics, public health adopted non-pharmaceutical interventions as a strategy to slow down the spread of the pandemic [21]. These interventions include social distancing measures

Keywords and phrases: SARS-CoV-2, COVID-19, non-pharmaceutical interventions, multi-scale modelling.

Team Complex Systems and Interactions, Central School of Casablanca, Casablanca, Morocco.

* Corresponding author: anass.bouchnita@centrale-casablanca.ma 
which aim to impose a minimum distance that has to be kept between individuals. Thus, reducing the chances of person-to-person contact. Other interventions minimise the chances of disease transmission during contact. This can be done by promoting hygiene practices, mask-wearing, and cough etiquette. The rest of non-pharmaceutical interventions decrease the chances of indirect transmission by frequently disinfecting public areas and products.

The first confirmed COVID-19 case in Morocco was reported on March 2, 2020. Since then, authorities have adopted several measures to slow down the propagation of the epidemic. First, international flights were gradually suspended. Next, school and university closures were mandated while opting for distance learning alternatives. A state of health emergency was declared on March 19, 2020 which limits the movement of the population to essentials. Finally, the use of face masks by individuals during movements was mandated on April 6,2020 . These measures will certainly slow the spread of COVID-19. However, the effect of each of them on the epidemic curve remains hard to quantify.

Mathematical modelling provides a tool to better understand the transmission dynamics of infectious diseases. Compartmental models are one of the most widely used to describe the dynamics of epidemics. In this framework, the changes in the populations of several classes of interacting individuals are described using ordinary differential equations (ODEs). Susceptible-infected-recovered (SIR) is one of the most commonly used types of compartmental models in epidemiology [2]. A recent SIR model which takes into consideration the effect of quarantine was formulated and used to estimate the basic reproduction number $\left(R_{0}\right)$ in China, Korea, and Italy [20]. Susceptible-exposed-infectious-recovered (SEIR) is another class of compartmental models that is used to simulate the transmission dynamics of diseases with a relatively long incubation period. These models were also used to estimate the basic reproduction number for COVID-19 [14, 17]. The advantage of compartmental models is that they can be studied both analytically and numerically.

Agent-based models can also be used to describe the transmission dynamics of infectious diseases. These models provide a more accurate description of the interactions that take place between individuals. Inter-agent communication can be implemented using a dynamic network $[1,5]$. These individual-based models provide an accurate description of the transmission dynamics that are specific for different regions and countries [6]. In a recent study, an individual-based model was used to assess the impact of non-pharmaceutical interventions on the COVID-19 outbreak in 11 European countries [7]. Social force models provide a more realistic description of the motion of individuals in crowds and under other various conditions [9]. A social force model was used to simulate the spread of a disease in an airplane [16]. Multi-scale modelling is a mechanism-based approach that integrates the available knowledge and data across several scales of space and time. As a result, dynamics of disease transmission are simulated within- and between-host organisms [8]. Thus, the response of each individual to the infection can depend on his immune system and health condition.

In this paper, we apply a previously developed model [4] to assess the impact of restricted population movement and mandatory mask-wearing on the transmission dynamics of COVID-19 in Morocco. The objective of this work is not to provide accurate predictions on the evolution of the epidemic but rather to understand and quantify the impact of the adopted strategy on disease propagation. To achieve this, we use a social force model to describe the random walk of individuals. We consider two modes of disease transmission. First, through direct person-to-person contact. Second, through contact with contaminated surfaces. Infected individuals do not develop symptoms until the end of the incubation period. They remain for a few days on the computational domain and then go into quarantine. The death probability of each individual agent depends on its age and health conditions. We begin by studying the impact of the restricted population movement and mandatory mask-wearing. We do this by reproducing the reported data for confirmed cases first. Then, we repeat the same simulation but without control measures and compare the obtained results.

\section{Multi-SCAle modelling of COVID-19 Transmission DYNAMICS IN MOROCCO}

In this work, we adapt a previously developed multi-scale model [4] to simulate the transmission dynamics of COVID-19 in Morocco. The model describes the spread of the disease in a population of individuals that move randomly in a square domain of $250 \times 250 \mathrm{~m}^{2}$. We introduce agents at random locations of the domain at the 
TABLE 1. Default values for the parameters of the social force model taken from [11].

\begin{tabular}{cccc}
\hline Parameter & Symbol & Value & Unit \\
\hline Time step & $\mathrm{d} t$ & $1 e^{-4}$ & $\mathrm{~h}$ \\
Population density & $\rho$ & 1000 & $\mathrm{ped} / \mathrm{km}^{2}$ \\
Walking speed & $\left\|v_{d}\right\|$ & $\mathrm{N}(1.34,0.26)$ & $\mathrm{m} \cdot \mathrm{s}^{-1}$ \\
Radius & $r_{i}$ & 0.23 & $\mathrm{~m}$ \\
Mass & $m_{i}$ & 83 & $\mathrm{~kg}$ \\
Relaxation time & $\tau_{i}$ & 0.5 & $\mathrm{~s}$ \\
\hline
\end{tabular}

beginning of each simulation and we consider that one agent is infected with COVID-19 and symptomatic. The model describes the movement of individual agents using a social force model. It considers both the modes of direct and indirect transmissions. We consider that infected individuals start transmitting the disease during the terminal stages of the incubation period. The mortality risk of each patient depends on its age and health conditions. We set periodic boundary conditions at the four boundaries to approximate larger systems.

The model is based on several assumptions. Therefore, it is not suitable to provide accurate predictions of the epidemic evolution in the next few weeks. Still, it can be used to gain a deeper understanding of the impact of restricted population movement and mask-wearing on the spread of the disease. The first assumption is that we simulate the transmission dynamics in a closed system of individuals. This is because of the suspended flights and closed borders. Children are not considered in the present study as they are not too much impacted by the virus. Furthermore, we assume that the only cause of death in the model is by COVID-19 during the simulated period to simplify the interpretation of the results. Finally, we consider only pre-symptomatic transmission but not asymptomatic one. This is because there are few reported cases of asymptomatic carriers who are not pre-symptomatic. We have shown a snapshot of a numerical simulation of the model in Figure 1A.

\subsection{Agent displacement}

We use a social force model [9] to describe the motion of each individual agent. The model was used previously to describe the movement of pedestrian in crowded areas [11]. We model each agent as a sphere particle which is subjected to several forces. We apply Newton's law to the center of each agent:

$$
\begin{gathered}
m_{i} \frac{\mathrm{d} v_{i}}{\mathrm{~d} t}=f_{i}^{\text {self }}+\xi_{i} \\
\frac{\mathrm{d} x_{i}}{\mathrm{~d} t}=v_{i},
\end{gathered}
$$

where $x_{i}$ is the displacement of the agent, $v_{i}$ is its velocity, $m_{i}$ is its mass, $\xi_{i}$ is a random perturbation and $f^{\text {self }}$ is the self-driven force defined by:

$$
f_{i}^{s e l f}=m_{i} \frac{v_{d, i}-v_{i}}{\tau_{i}}
$$

$v_{d, i}$ is the desired velocity at which the $i$ th pedestrian wants to move. We consider that agents tend to move to random directions, the amplitude of the desired speed is chosen to be following a normal distribution with an average of $1.34 \mathrm{~m} . \mathrm{s}^{-1}\left(\simeq 5 \mathrm{~km} \mathrm{~h}^{-1}\right)$ and a standard deviation of $0.26 \mathrm{~m} \cdot \mathrm{s}^{-1}, \tau_{i}$ is a relaxation time. Numerical values of parameters are provided in Table 1. 
TABLE 2. Values of the considered COVID-19 characteristics in the model.

\begin{tabular}{ccc}
\hline Characteristic time & Median (standard deviation) & Distribution and reference \\
\hline Incubation period & $5.1(3.1)$ days & log-normal distribution [12] \\
Pre-symtomatic transmission & 1 day & uniform [22] \\
Onset to quarantine & $3.9(0.5)$ days & normal distribution [13] \\
Quarantine to discharge & $20.2(2)$ days & normal distribution [13] \\
Quarantine to death & $13(1)$ days & normal distribution [13] \\
\hline
\end{tabular}

\subsection{Modes of disease transmission}

We consider that contagious individuals can transmit the disease by two means. First, they can infect susceptible individuals if they get into direct with them. We consider that each susceptible individual have a probability $p_{d}$ to contract the virus upon direct contact with a contagious agent. Second, infectious contaminate neighboring surfaces by secreting droplets which contain the virus. These surfaces can subsequently transmit the virus to susceptible individuals. We describe the concentration of stable SARS-CoV-2 on hard surfaces as follows:

$$
\frac{\partial C}{\partial t}=W_{i}-\sigma C
$$

where $W_{i}$ is the secretion rate of the virus by infected individuals and $\sigma$ is the degradation rate of stable SARS-CoV-2. The probability of viral infection by touching hard surfaces is estimated as:

$$
p_{\text {in }}=\lambda C\left(x_{i}\right) \text {, }
$$

where $\lambda$ is a positive constant taken smaller than one. Note that for indirect transmission to take place, the individual has to touch the contaminated surface and then his or her face before washing their hands. Therefore, we only evaluate the possibility of indirect transmission once each day for each agent at a random moment of the day.

\subsection{Clinical course of infected patients}

Infected agents do not develop symptoms until the end of the incubation period. We consider that they start transmitting the virus a day before the onset of symptoms [22]. We have studied the effect of pre-symptomatic transmission on the transmission dynamics of COVID-19 in a previous study [4]. The median value for the incubation period is 5.1 days and we use a log-normal distribution to sample this period for each agent $[12,13]$. After the development of symptoms, infected agents remain for a few days in the computational domain. Then, they get isolated and go into quarantine at home or in a hospital. In this period of quarantine, the patient stops moving in the computational domain and interacting with other agents. This phase can have two outcomes: the patient can either die or survive. If he or she survives, then they start moving as before and they become immunized to new infections. Median values for characteristic periods and the distribution used for their sampling them are given in Table 2. We represent the evolution of infected individuals in Figure 1B.

\subsection{Demographic characteristics and mortality risks}

We consider that the death probability for each patient depends on its characteristics and in particular age and pre-existing risk factors. We represent the age-structure as a distribution function and we use it to sample the age of each patient. We consider that the age of each agent determines the risk of its COVID-19-related mortality as represented in Table 3 [18]. 
A)
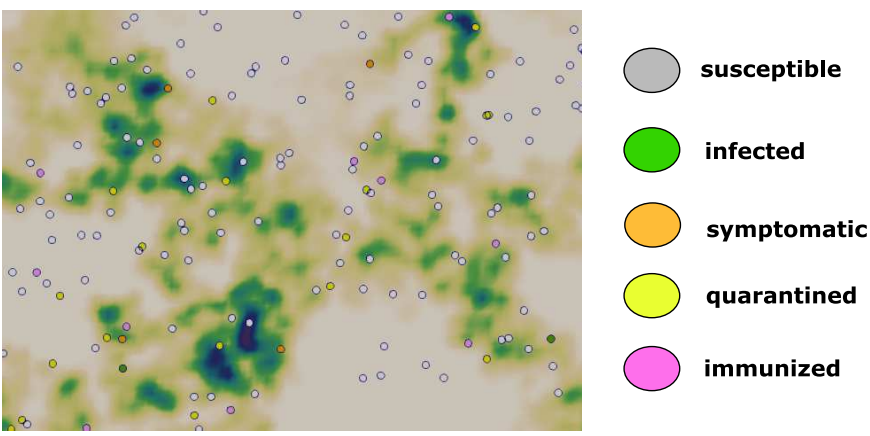

B)

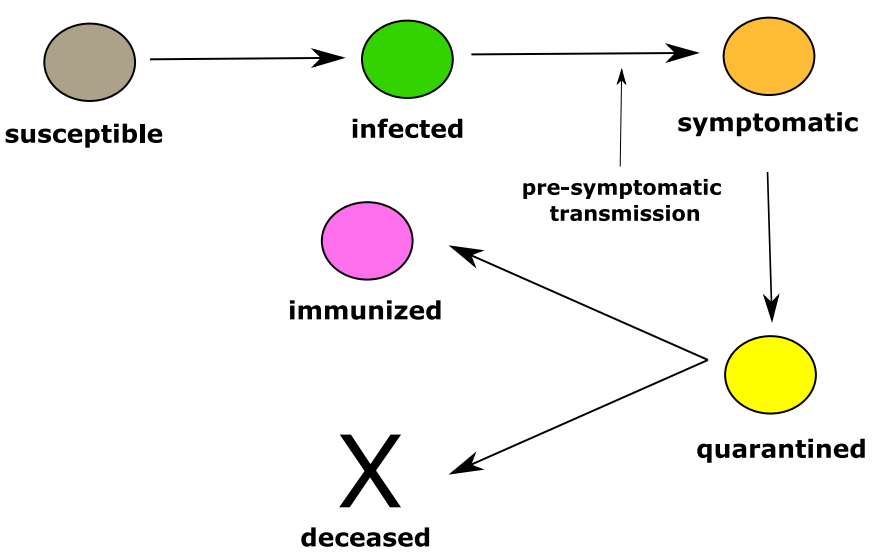

Figure 1. (A) Snapshot of a numerical simulation of the model. Spheres represent agents moving in a square section of $250 \mathrm{~m} \times 250 \mathrm{~m}$. The color of each agent represents the class to which its belong: white for susceptible and immunized, green for infected, orange for symptomatic, and yellow for quarantined. The concentration of stable SARS-CoV-2 on hard surfaces is represented using the gradient of the green color. (B) The clinical course of COVID-19 patients in the model.

TABLE 3. The prevalence of age groups in the model and the corresponding mortality risk for each group.

\begin{tabular}{ccc}
\hline Age group & Prevalence in the model & Death probability \\
\hline $18-29$ & $19.24 \%$ & $0.2 \%$ \\
$30-39$ & $19.24 \%$ & $0.2 \%$ \\
$40-49$ & $19.24 \%$ & $0.4 \%$ \\
$50-59$ & $19.24 \%$ & $1.3 \%$ \\
$60-69$ & $14.57 \%$ & $3.6 \%$ \\
$70-79$ & $6.08 \%$ & $8 \%$ \\
$80-90$ & $2.39 \%$ & $14.8 \%$ \\
\hline
\end{tabular}


TABLE 4. The prevalence of COVID-19 mortality risk factors in the model and the corresponding death probability.

\begin{tabular}{ccc}
\hline Risk factor & Sampling probability in the model & Death probability \\
\hline Cardiovascular diseases & $25 \%$ & $13.2 \%$ \\
Diabetes & $8 \%$ & $9.2 \%$ \\
High blood pressure & $25 \%$ & $8.4 \%$ \\
Chronic respiratory diseases & $5 \%$ & $8 \%$ \\
Cancer & $0.57 \%$ & $7.6 \%$ \\
\hline
\end{tabular}

An individual can also have one or many of the pre-existing risk factors which increase the mortality risk of COVID-19. These risk factors include chronic respiratory diseases, cardiovascular diseases, elevated blood pressure, diabetes, and cancer. We consider that the prevalence of these health conditions to be similar to the one observed in society. Table 4 provides the prevalence of these conditions and the corresponding death probability taken from a World Health Organization report [21].

\subsection{Computer implementation}

The model is implemented using the $\mathrm{C}++$ language. To ensure code modularity, we have used an objectoriented programming (OOP) architecture. The average CPU time of a numerical simulation of 120 days of transmission dynamics is around $3.4 \mathrm{~h}$ in a computer with four cores and $6 \mathrm{~Gb}$ of RAM. The post-processing of the results was done using the ParaView software and python scripts. The code can be accessed at: https://github.com/MPS7/SIM-CoV

\section{Results}

\subsection{Numerical simulation of COVID-19 transmission dynamics in Morocco}

We begin by studying the dynamics of COVID-19 transmission in Morocco. To achieve this, we fit two of the model parameters to reproduce the reported data on cumulative cases as of April 10, 2020 [15]. These two parameters are the probabilities of direct transmission $\left(p_{d}\right)$ and indirect transmission $\left(p_{\text {ind }}\right)$. We consider that the state of health emergency imposed on March 20, 2020, corresponds to the immobilization of $60 \%$ of the population. This is because the curfew begins each day from $6 \mathrm{pm}$ to $6 \mathrm{am}$. Furthermore, Medical and banking personnel as well as logistics workers handling consumer goods, agricultural produce, pharmaceutical products, medical supplies and equipment, post are exempt from curfew restrictions. The mandatory wearing of face masks was imposed on April 6, 2020. Individuals were required to wear face masks if they move outside home. We model this measure by reducing the probability of transmitting or contracting the disease for mobile agents by half. Furthermore, we consider that these mobile agents have a lower production rate of the virus. Hence, we consider that the value of SARS-CoV-2 production rate $\left(W_{i}\right)$ for mobile agents is half the normal value for immobilized individuals.

Restricting the movement of the population results in a reduction of the probability of person-to-person contact. The wearing of face masks reduces the probabilities of direct and indirect transmission. Virus transmission begins two days after the introduction of the first case due to pre-symptomatic transmission. A few symptomatic individuals transmit the disease when the restricted movement measure starts. Immobilized agents contaminate neighbouring surfaces with a higher SARS-CoV-2 concentration than mobile individuals. The peak of the epidemic, which corresponds to the stabilization of the daily number of new cases, is reached on April 21, 2020. At this moment, a portion of the individuals is infected and a part of them is quarantined. The spread of the disease is stopped when all infected individuals are quarantined and the concentration of the virus on the stable surfaces reaches zero. The results of a numerical simulation for the transmission dynamics of COVID-19 in Morocco are shown in Figure 2. According to the model predictions, the peak is reached on April 21, 2020. The 
i) March 9, 2020

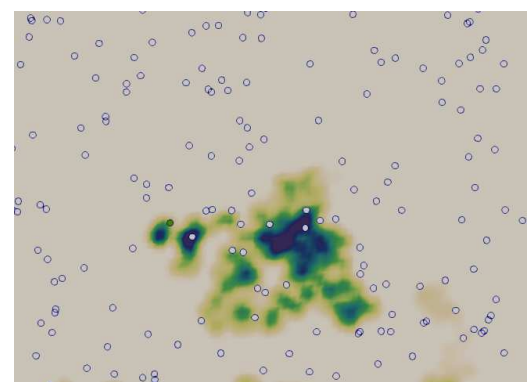

iii) April 6, 2020

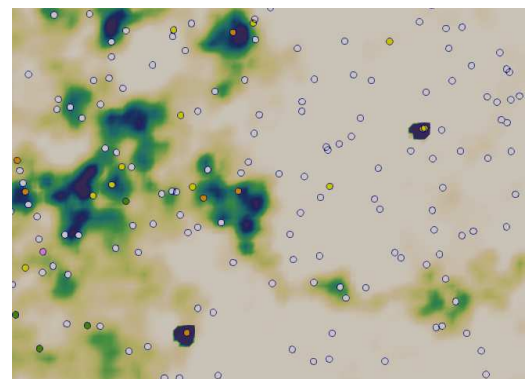

v) May 15, 2020

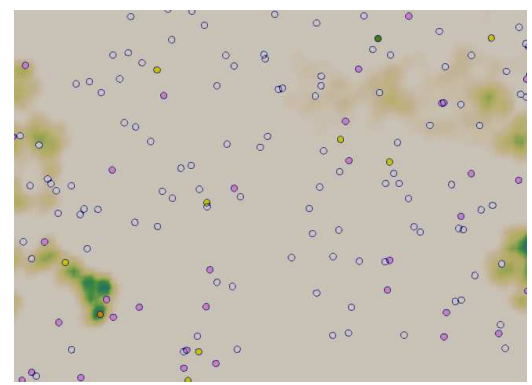

ii) March 20, 2020

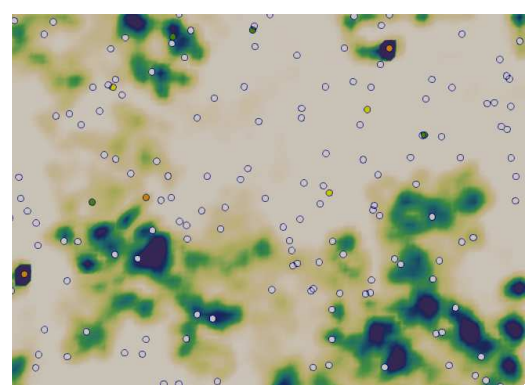

iv) April 21, 2020

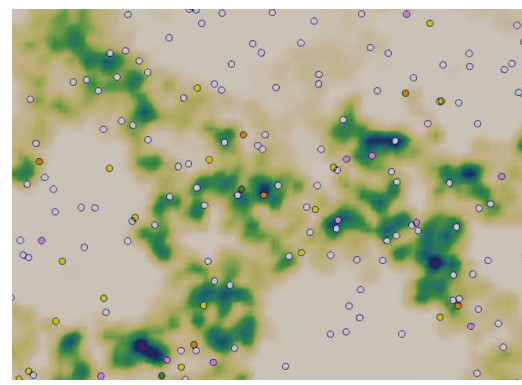

vi) June 15, 2020

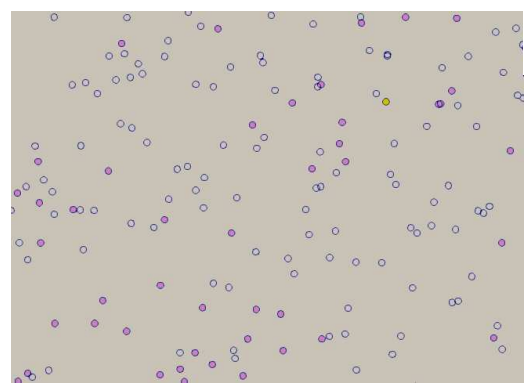

FigURE 2. Snapshots of a numerical simulation corresponding to the COVID-19 transmission dynamics in Morocco. i) A few days after the confirmation of the first case. Some of the infected cases started transmitting the virus before they develop symptoms. ii) The state of a health emergency is imposed. A few agents are infected. Some are quarantined and one is symptomatic. iii) The situation when the mandatory wearing of face masks was imposed. Some cases are infected. Immobilized agents can contaminate local surfaces with a high concentration of the virus upon infection. iv) The peak of the epidemic. A significant fraction of agents is infected. v) The spread of the disease is over. All active cases are quarantined and cannot transmit the virus. vi) The epidemic is resolved and a few remaining cases are quarantined. A portion of the population is immunized to the disease.

cumulative fraction of infected cases out of detected susceptible individuals is $30.8 \%$. The percent of deceased agents is $2.4 \%$ which corresponds to a case fatality rate (CFR) of $7.7 \%$. The last case of infection takes place on May 16, 2020. However, it takes one additional month for the number of active infected cases to reach zero. In the remainder of the paper, we refer to this strategy as scenario III. 
A)

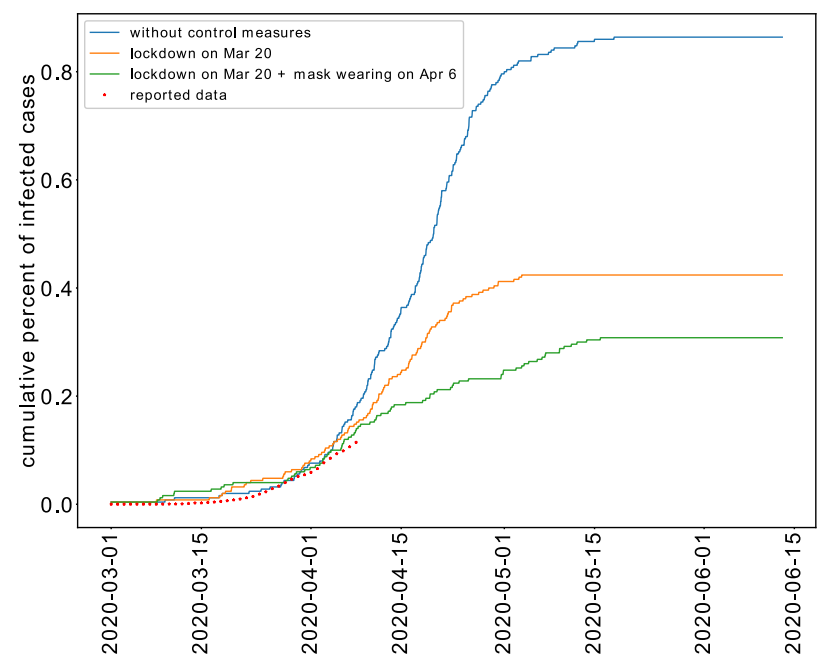

B)

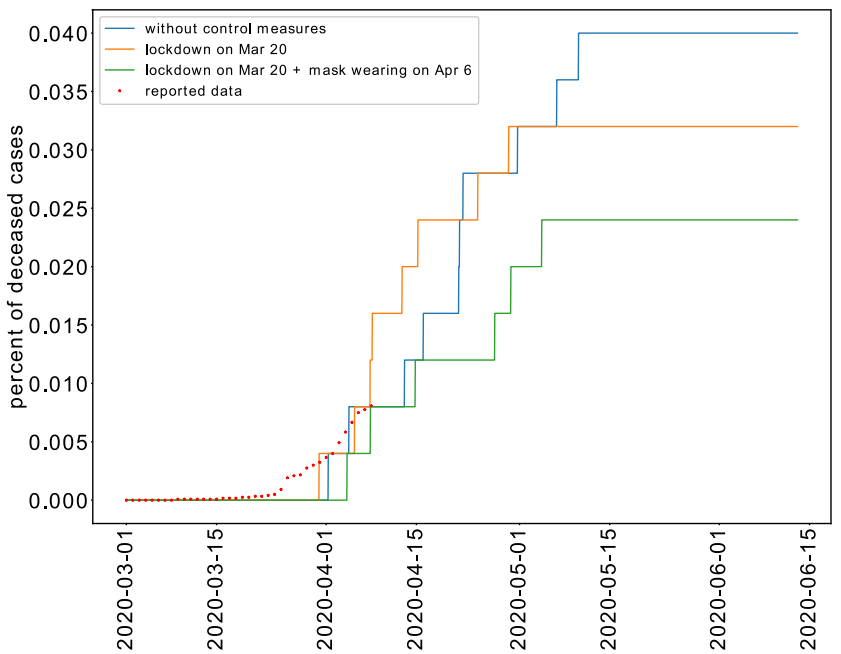

Figure 3. A) The cumulative percent of infected cases over time according to the model predictions for three different epidemic control strategies. The reported data as of April 10, 2020 is shown as well. B) The total percent of deceased agents according to the model predictions and the reported data [15] as of April 10, 2020.

\subsection{Restricted movement of population and mandatory wearing of face masks reduce the total number of COVID-19 cases by $64 \%$}

We consider the same parameters of the previous simulation and we lift the measure of mandatory wearing of face masks. We refer to this strategy as scenario II. In this case, the cumulative percent of infected cases increases to $42.4 \%$. The cumulative percent of deaths is $3.2 \%$ which corresponds to a CFR equal to $7.5 \%$. If we do not consider the restriction of movement, the cumulative percent of infected cases reaches $86.4 \%$. This shows that the adopted measures would reduce the cumulative percent of COVID-19 by $64 \%$. If no measures were adopted, the cumulative percent of deceased patients would reach $4 \%$ of the detected susceptible population. We designate this situation as scenario I. The effect of the adopted measures on the percent of infected cases is presented on Figure 3 and the cumulative percent of deceased agents for the three scenarios is show in Figure 3B.

To better understand the dynamics of COVID-19 transmission, we study the evolution of the active percents of infected and symptomatic agents for each of these three cases. In the scenario I, the epidemic peak is reached on May 3, 2020. The last infection takes place on May 16, 2020, and the epidemic is resolved on June 14, 2020. When we consider that the movement of population is restricted, the peak of the epidemic is reached on April 25, 2020. The last case is reported on May 05, 2020 and the epidemic ends on June 03, 2020. We have represented the evolution of the active percent of infected and symptomatic agents in Figure 4. The results of the three simulations are summarized in Table 5.

Among the goals of adopting non-pharmacological interventions is to reduce the burden on the health care system. Indeed, reducing the chances of disease transmission decreases the active number of individuals who require treatment. The model can be used to estimate the burden on the health system. Indeed, we compute the active percent of quarantined individuals for the three scenarios (Fig. 5). In the absence of control measures, the percent of quarantined agents is expected to reach $38 \%$ during the peak of the epidemic. When we restrict the movement of the population, the maximum percent of quarantined percent becomes $15.5 \%$. Finally, when we consider the mandatory wearing of masks in addition to the restricted movement of the population, the maximum percent of quarantined individuals falls to $10.3 \%$. 
A)

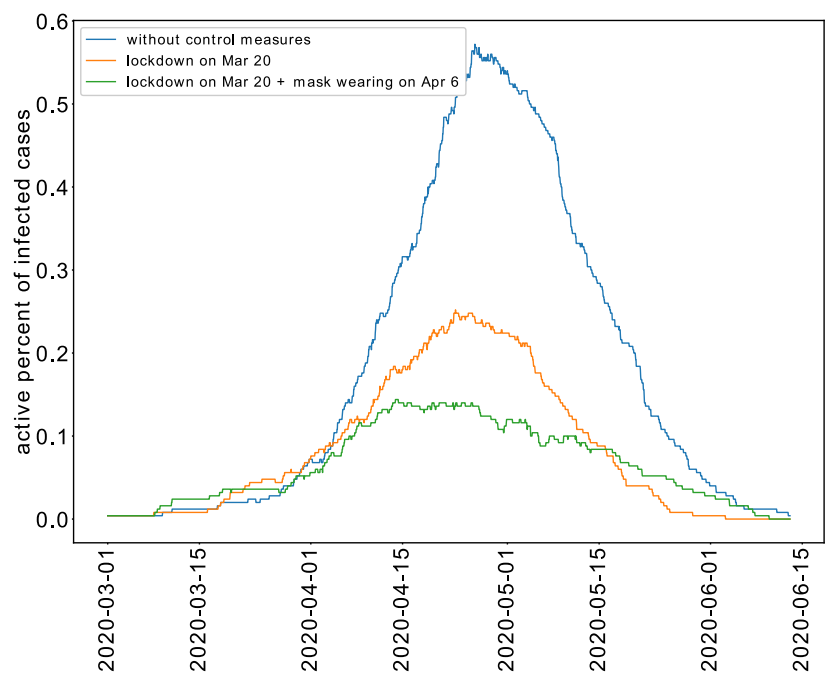

B)

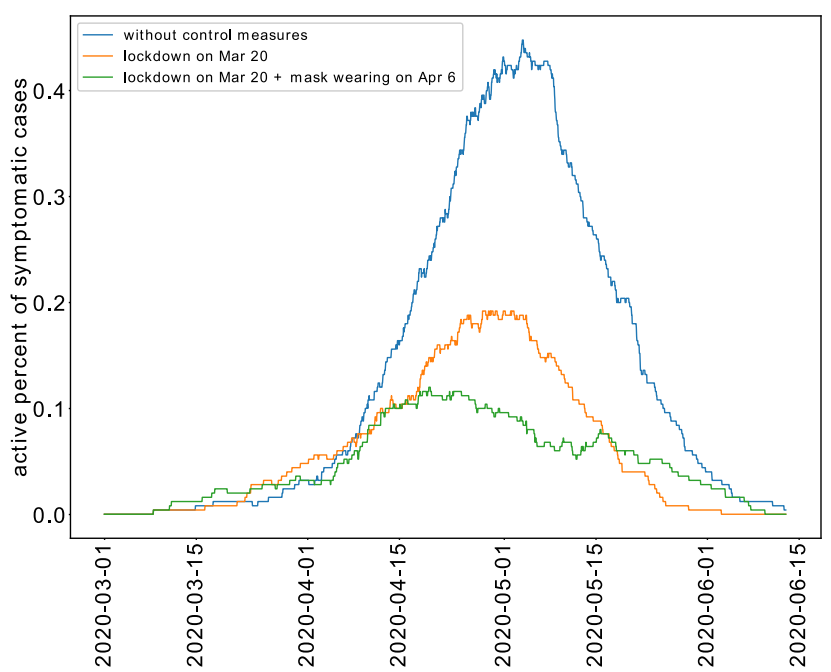

Figure 4 . The active percent of infected cases (A) and the active percent of symptomatic cases (B) for three different scenarios.

TABLE 5. A summary of the model predictions for the three scenarios. The dates are given in the $\mathrm{dd} / \mathrm{mm} /$ yyyy format.

\begin{tabular}{cccccc}
\hline Scenario & Total cases & Percent of deaths & Peak date & Last case & Epidemic end \\
\hline I- Without control measures & $86.4 \%$ & $4 \%$ & $03 / 05 / 2020$ & $16 / 05 / 2020$ & $14 / 06 / 2020$ \\
II- Restricted movement & $42.4 \%$ & $3.2 \%$ & $25 / 04 / 2020$ & $05 / 05 / 2020$ & $03 / 06 / 2020$ \\
$\begin{array}{c}\text { III- Restricted movement } \\
\text { + mask wearing }\end{array}$ & $30.8 \%$ & $2.4 \%$ & $21 / 04 / 2020$ & $15 / 05 / 2020$ & $12 / 06 / 2020$ \\
\hline
\end{tabular}

\subsection{Restricted movement and mandatory mask-wearing increase the incidence of indirect transmission}

One of the main features of SARS-CoV-2 is its relatively high half-life time on hard surfaces. As a result, the chances of indirect transmission of the disease can be considerable. We represent the average concentration of the virus over time in the computational domain for the three scenarios in Figure 6. As expected, the concentration of the viable virus is higher when the control measures are not adopted. We calculate the ratio of indirect transmissions as the percent of indirect transmissions out of the total number of infections. We present the relative incidence of indirect transmissions for the three cases in Table 6 . When the control measures are considered, the rate of indirect transmission increases despite the lower concentration of the virus. Indeed, the restricted movement of the population and the mandatory wearing of masks mainly reduce the chances of person-to-person contact and direct transmission.

\subsection{Lifting control measures before the end of the epidemic results in the resurgence of the outbreak}

Limiting the movement of the population has several economic and social impacts. Therefore, it is important to be able to relax or completely lift epidemic control measures as soon as possible. We investigate the effects of lifting non-pharmaceutic interventions during the final phase of the epidemic. We consider that the control measures are lifting on May 15, 2020. At this moment of the simulation, there is still five symptomatic agents 


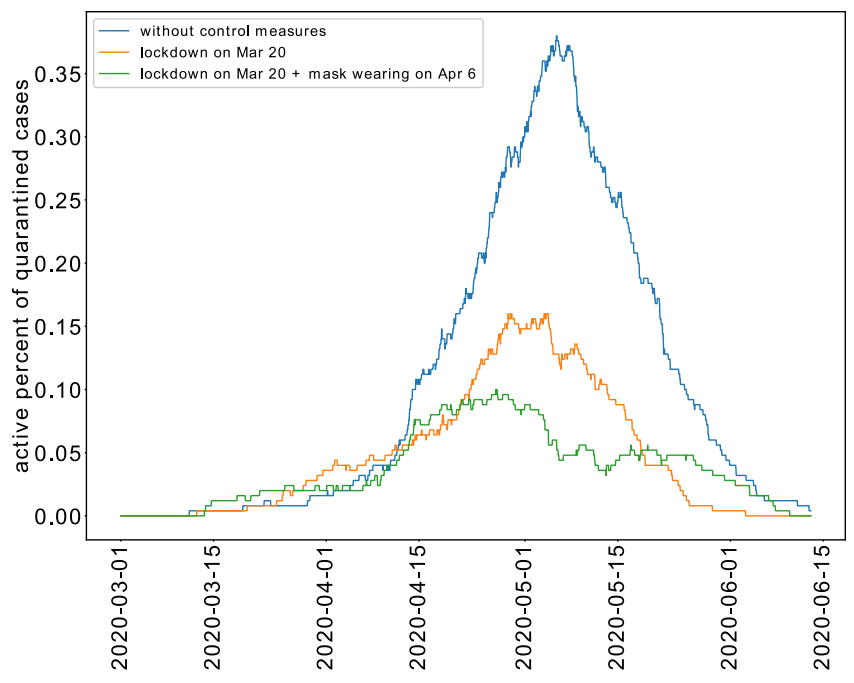

Figure 5. The active percent of quarantined cases for the three different scenarios.

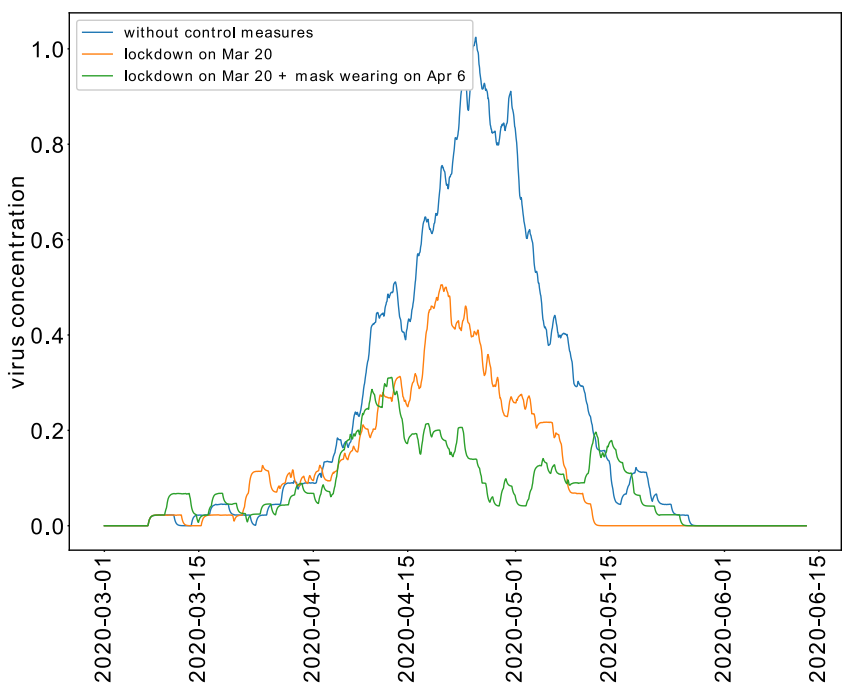

FiguRE 6 . The average concentration of stable SARS-CoV-2 in the computational domain over time.

TABLE 6 . The rate of indirect disease transmission for each case.

\begin{tabular}{ccc}
\hline Scenario & $\begin{array}{c}\text { Number of } \\
\text { indirect transmissions }\end{array}$ & $\begin{array}{c}\text { Ratio of } \\
\text { indirect transmissions }\end{array}$ \\
\hline I- Without control measures & 45 & $17.9 \%$ \\
II- Limited movement of the population & 28 & $20.5 \%$ \\
III- Limited movement of the population & 20 & $33.7 \%$ \\
+ mandatory mask-wearing & & \\
\hline
\end{tabular}




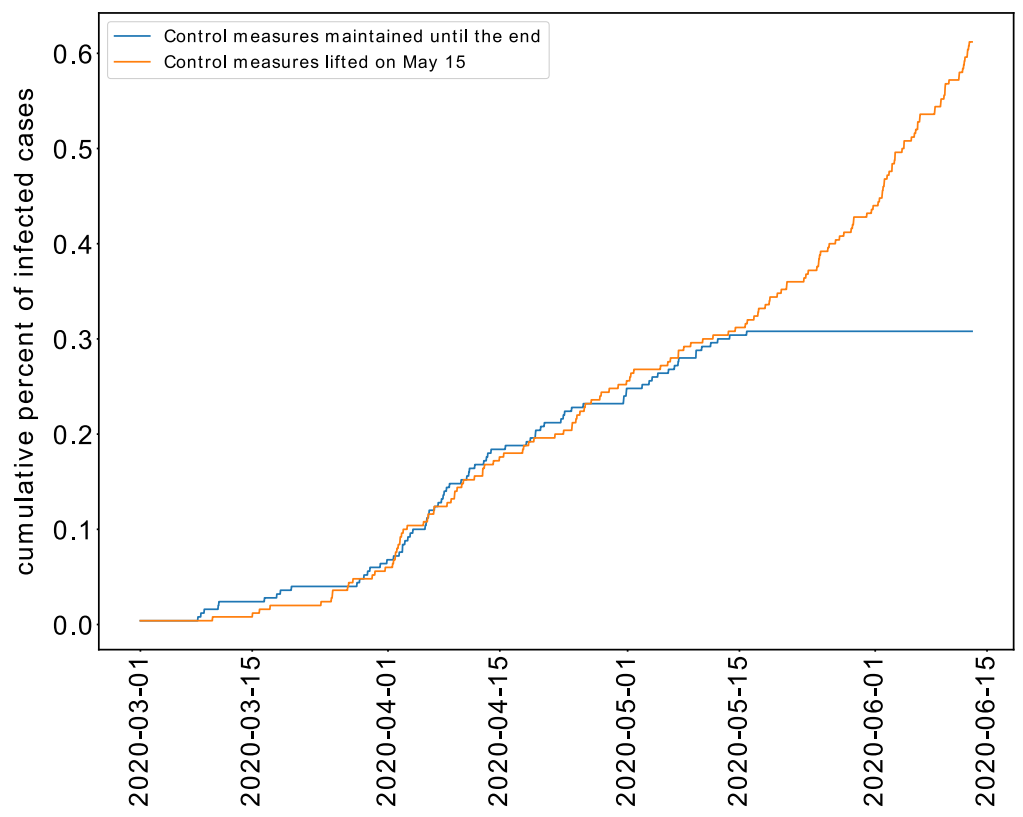

Figure 7. The cumulative percent of infected cases when control measures are maintained until the end $v s$. when they are lifted before the end of the epidemic.

that are not quarantined yet and the surfaces are still contaminated. As a result, we observe that the cumulative percent of infected individuals do not stabilize but starts growing exponentially again (Fig. 7). Therefore, lifting control measures should only take place when all infected cases are quarantined and when the concentration of the virus on the computational domain reaches zero.

\section{Discussion}

In this work, we apply a previously developed multi-scale model [4] to quantify the effect of non-pharmaceutical interventions on the transmission dynamics of COVID-19 in Morocco. The model uses a social force model to describe the motion of individuals which makes it suitable for the simulation of the impact of NPIs on the movement of the population. It considers both modes of direct and indirect transmission. Furthermore, it takes into account pre-symptomatic transmission which characterizes COVID-19 infection. We assign an age to each agent which determines its mortality risk. Agents can also have one or more of the mortality risk factors. The multi-scale structure of the model allowed us to integrate the available data on COVID-19 transmission and infection dynamics at the individual and population levels. According to the model predictions, the adoption of epidemic control measures would reduce the cumulative number of infected cases by $64 \%$. This is because the restriction of movement decreases the probability of direct contact while the wearing of masks reduces the probabilities of direct and indirect disease transmission. The obtained results suggest that the adopted measures would reduce the number of infected cases but they would not shorten the duration of the epidemic. Furthermore, Numerical simulations reveal that these measures reduce the concentration of stable virus on the computational domain. However, the rate of indirect transmission increases when the movement of the population is limited and the wearing of masks is mandatory. This suggests that other measures that target the mode of indirect transmission will have a significant impact and will further reduce the number of infected cases. Indeed, the disinfection of public areas would reduce virus concentration and decrease the incidence of indirect transmissions. Finally, the model suggests that the early lifting of NPIs could result in a resurgence of 
the epidemic. Therefore, it is important to wait until all infected cases are quarantined and that stable SARS$\mathrm{CoV}-2$ on hard surfaces has decayed. We will study the relaxation strategies of epidemic control measures in a forthcoming work.

The goal of this study is to assess the impact of NPIs on the transmission dynamics of COVID-19 in Morocco. It is not to give accurate predictions on the evolution of the epidemic situation. Indeed, the model relies on several assumptions which can be hard to verify in reality. First, the model does not consider the effect of children in transmitting the virus. This assumption was considered because children are supposed to be staying at home because of school closures. Second, we assume that patients who survived the disease become immune to new infections. However, the development of immunity to COVID-19 upon infection is still under investigation. Second, the model assumes that all cases of asymptomatic transmission are pre-symptomatic. In the absence of sufficient data on the infection dynamics in asymptomatic cases, we consider all susceptible individuals to be potentially symptomatic. Third, the model considers a relatively low number of agents. The use of a small time step in numerical simulation is necessary to track all contacts between individuals. This significantly increases the CPU time. Finally, data concerning the mortality of COVID-19 were taken from clinical studies conducted in China. In a forthcoming work, we will develop an immuno-epidemiological model by coupling the present model with another model describing the host-specific immune response of infected individuals [3].

\section{REFERENCES}

[1] M. Ajelli, B. Gonçalves, D. Balcan, V. Colizza, H. Hu, J.J. Ramasco, S. Merler and A. Vespignani, Comparing large-scale computational approaches to epidemic modeling: agent-based versus structured metapopulation models. BMC Infect. Dis. 10 (2010) 190.

[2] L.J. Allen, Some discrete-time si, sir, and sis epidemic models. Math. Biosci. 124 (1994) 83-105.

[3] M. Banerjee, A. Tokarev and V. Volpert, Immuno-epidemiological model of two-stage epidemic growth. Preprint arXiv:2003.14152 (2020).

[4] A. Bouchnita and A. Jebrane, A hybrid multi-scale model of covid-19 transmission dynamics to assess the potential of nonpharmaceutical interventions. Preprint medRxiv 20054460v1 (2020).

[5] W. Dong, K. Heller and A.S. Pentland, Modeling infection with multi-agent dynamics, in International Conference on Social Computing, Behavioral-Cultural Modeling, and Prediction. Springer, Berlin (2012) 172-179.

[6] N.M. Ferguson, D.A. Cummings, C. Fraser, J.C. Cajka, P.C. Cooley and D.S. Burke, Strategies for mitigating an influenza pandemic. Nature 442 (2006) 448-452.

[7] S. Flaxman, S. Mishra, A. Gandy, H. Unwin, H. Coupland, T. Mellan, H. Zhu, T. Berah, J. Eaton, P. Perez Guzman, et al. Report 13: Estimating the number of infections and the impact of non-pharmaceutical interventions on covid-19 in 11 European countries (2020).

[8] W. Garira, A complete categorization of multiscale models of infectious disease systems. J. Biol. Dyn. 11 (2017) $378-435$.

[9] D. Helbing and P. Molnar, Social force model for pedestrian dynamics. Phys. Rev. E 51 (1995) 4282.

[10] D.S. Hui, E.I. Azhar, T.A. Madani, F. Ntoumi, R. Kock, O. Dar, G. Ippolito, T.D. Mchugh, Z.A. Memish, C. Drosten, et al. The continuing 2019-ncov epidemic threat of novel coronaviruses to global health-the latest 2019 novel coronavirus outbreak in Wuhan, China. Int. J. Infect. Dis. 91 (2020) 264.

[11] B. Kabalan, P. Argoul, A. Jebrane, G. Cumunel and S. Erlicher, A crowd movement model for pedestrian flow through bottlenecks. Ann. Solid Struct. Mech. 8 (2016) 1-15.

[12] S.A. Lauer, K.H. Grantz, Q. Bi, F.K. Jones, Q. Zheng, H.R. Meredith, A.S. Azman, N.G. Reich and J. Lessler, The incubation period of coronavirus disease 2019 (covid-19) from publicly reported confirmed cases: estimation and application. Ann. Internal Med. $172(2020) 577-582$.

[13] N.M. Linton, T. Kobayashi, Y. Yang, K. Hayashi, A.R. Akhmetzhanov, S.-m. Jung, B. Yuan, R. Kinoshita and H. Nishiura, Incubation period and other epidemiological characteristics of 2019 novel coronavirus infections with right truncation: a statistical analysis of publicly available case data. J. Clin. Med. 9 (2020) 538.

[14] Y. Liu, A.A. Gayle, A. Wilder-Smith and J. Rocklöv, The reproductive number of covid-19 is higher compared to sars coronavirus. J. Travel Med. 27 (2020) taaa021.

[15] M. Ministère de la santé, Bulletin hebdomadaire covid 19. Available at: http://www.covidmaroc.ma/ (2020).

[16] S. Namilae, A. Srinivasan, A. Mubayi, M. Scotch and R. Pahle, Self-propelled pedestrian dynamics model: Application to passenger movement and infection propagation in airplanes. Physica A 465 (2017) 248-260.

[17] J. Rocklöv, H. Sjödin and A. Wilder-Smith, Covid-19 outbreak on the diamond princess cruise ship: estimating the epidemic potential and effectiveness of public health countermeasures. To appear in: J. Travel Medicine (2020) taaa030.

[18] V. Surveillances, The epidemiological characteristics of an outbreak of 2019 novel coronavirus diseases (covid-19)-China, 2020. China CDC Weekly 2 (2020) 113-122. 
[19] N. van Doremalen, T. Bushmaker, D.H. Morris, M.G. Holbrook, A. Gamble, B.N. Williamson, A. Tamin, J.L. Harcourt, N.J. Thornburg, S.I. Gerber, et al., Aerosol and surface stability of sars-cov-2 as compared with sars-cov-1. New Engl. J. Med. 382 (2020) 1564-1567.

[20] V. Volpert, M. Banerjee and S. Petrovskii, On a quarantine model of coronavirus infection and data analysis. MMNP $\mathbf{1 5}$ (2020) 24.

[21] World Health Organization, Coronavirus disease 2019 (covid-19) situation report-41.

[22] World Health Organization, Coronavirus disease 2019 (covid-19) situation report-73. 